\title{
Rational Term Rewriting ${ }^{\star}$
}

\author{
A. Corradini ${ }^{1}$ and F. Gadducci ${ }^{2}$ \\ 1 Università di Pisa, Dipartimento di Informatica, Corso Italia 40, I-56214 Pisa, Italy \\ (andrea@i.unipi.it). \\ 2 TUB, Fachbereich 13 Informatik, Franklinstraße 28/29, D-10587 Berlin, Germany \\ (gfabio@cs.tu-berlin.de).
}

\begin{abstract}
Rational terms (possibly infinite terms with finitely many subterms) can be represented in a finite way via $\mu$-terms, that is, terms over a signature extended with self-instantiation operators. For example, $f^{\omega}=f\left(f(f(\ldots))\right.$ ) can be represented as $\mu_{x} . f(x)$ (or also as $\mu_{x} . f(f(x))$, $\left.f\left(\mu_{x} . f(x)\right), \ldots\right)$. Now, if we reduce a $\mu$-term $t$ to $s$ via a rewriting rule using standard notions of the theory of Term Rewriting Systems, how are the rational terms corresponding to $t$ and to $s$ related?

We answer to this question in a satisfactory way, resorting to the definition of infinite parallel rewriting proposed in [7]. We also provide a simple, algebraic description of $\mu$-term rewriting through a variation of Meseguer's Rewriting Logic formalism.
\end{abstract}

\section{Introduction}

Rational terms are possibly infinite terms with a finite set of subterms. They show up in a natural way in Theoretical Computer Science whenever some finite cyclic structures are of concern (for example data flow diagrams, cyclic term graphs, or process algebras with recursion), and one desires to abstract out from the "degree of folding" of such structures, intuitively identifying those that denote the same infinitary behaviour.

For example, the $\mu$-term $t_{1}=\mu_{x}$.ite $\left(B, \operatorname{seq}\left(C_{1}, x\right), C_{2}\right)$ can be used as a linear representation of a flow chart intended to model the structure of a while loop using the if-then-else (ite) and the sequentialization (seq) statements, where the boolean condition $B$ and the statements $C_{1}$ and $C_{2}$ are left unspecified. As stressed in [20], the intended meaning of the operator $\mu_{x}$, when applied to a term $t[x]$ with $x$ free, is of constraining the instantiation of $x$ in $t$ to $\mu_{x} . t$ only; thus $\mu_{x}$ can be considered as a self-instantiation operator. By performing this selfinstantiation once in $t_{1}$, we get $t_{2}=i \operatorname{te}\left(B, \operatorname{seq}\left(C_{1}, \mu_{x} . i t e\left(B, \operatorname{seq}\left(C_{1}, x\right), C_{2}\right)\right), C_{2}\right)$. Now, both $t_{1}$ and $t_{2}$ can be seen as a finite representation of the same infinite, rational term $i \operatorname{te}\left(B, \operatorname{seq}\left(C_{1}, \operatorname{ite}\left(B, \operatorname{seq}\left(C_{1}, \operatorname{ite}\left(B, \operatorname{seq}\left(C_{1}, \ldots\right), C_{2}\right)\right), C_{2}\right)\right), C_{2}\right)$, which, in turn, can be regarded as a representative of the equivalence class of $\mu$-terms containing $t_{1}$ and $t_{2}$. ¿From a computational viewpoint, rational terms are clearly

\footnotetext{
* Research partly supported by the EC TMR Network GETGRATS (General Theory of Graph Transformation Systems) through the Dipartimento di Informatica of Pisa and the Technical University of Berlin.
} 
a very interesting subclass of infinite terms, because they have a finitary representation; usually, however, this is not unique.

Infinitary extensions of Term Rewriting have been considered by various authors during the last decade $[12,11,15,16,7,20,21,22,9,8]$. Most of those contributions are concerned with the study of the rewriting relation induced by a set of finite term rules on infinite terms, presenting results about the existence of normal forms (possibly reachable after $\omega$ steps), confluence and so on. Only a few of them, namely [20, 21, 8], focus on the subclass of rational terms, regarded essentially as the semantics of some finite but possibly cyclic structures (term graphs or $\mu$-terms).

The goal of this paper is to provide a solid mathematical basis for the theory of rational term rewriting. One main requisite for us is that such a theory must provide a "clean" semantics for the rewriting of the finitary representations of rational terms. This is a not completely trivial task, as shown by the following two simple examples which make use of $\mu$-terms, the finitary representation of rational terms that we shall use along the paper.

Let $t$ be the $\mu$-term $t=\mu_{x} f(x)$, representing the rational term $f^{\omega} \stackrel{\text { def }}{=}$ $f(f(f(\ldots)))$, and let $R: f(y) \rightarrow g(y)$ be a term rewriting rule. Unlike for example [20], we insist that in our theory it should be possible to apply $R$ to $t$, obtaining, quite obviously, the reduction $\mu_{x} \cdot f(x) \rightarrow_{R} \mu_{x} \cdot g(x)$. If we consider the associated rational terms, this apparently innocuous rewriting step requires some infinitary extension of the theory of term rewriting, because there are infinitely many occurrences of $f$ in $f^{\omega}$, and all of them have to be changed to $g$ : in fact, the $\mu$-term $\mu_{x} . g(x)$ represents $g^{\omega}$.

There are two possible infinitary extensions of term rewriting that allow to formalize such a phenomenon. Using the theory of transfinite rewriting of [22] (and adopted by most of the papers mentioned above), one obtains $g^{\omega}$ as the limit (in the standard complete metric space of infinite terms [1]) of the infinite (Cauchy) sequence of reductions $f^{\omega} \rightarrow_{R} g\left(f^{\omega}\right) \rightarrow_{R} g\left(g\left(f^{\omega}\right)\right) \leadsto g^{\omega}$. Using instead the infinite parallel rewriting of [7], $g^{\omega}$ is obtained in a single reduction step by replacing in parallel all the occurrences of $f$ in $f^{\omega}$ by $g$ : this kind of reduction is defined using standard completion techniques that exploit the CPO structure of possibly partial, possibly infinite terms [19].

And what about the application of the "collapsing" rule $R^{\prime}: g(y) \rightarrow y$ to $\mu_{x} . g(x)$ ? There is no apparent reason to forbid it, and one would expect to obtain the reduction $\mu_{x} . g(x) \rightarrow_{R^{\prime}} \mu_{x} . x$. Considering the corresponding rational terms, by applying the theory of [22] we have that since $g^{\omega} \rightarrow_{R^{\prime}} g^{\omega}$, the limit of infinitely many such reductions cannot be different from $g^{\omega}, 3$ which is not related at all to $\mu_{x} . x$. Using the infinite parallel rewriting of [7], instead, we have that $g^{\omega}$ rewrites to $\perp$, the bottom element of the CPO of terms, and $\perp$ is indeed the canonical interpretation of the $\mu$-term $\mu_{x} . x$, according to the Iteration Algebras framework [3]. An infinite term made of infinitely many nested redexes of collapsing rules (as $g^{\omega}$ in this example) will be called a "hypercollapsing

${ }^{3}$ Actually such a derivation is not strongly convergent, and thus it is not considered admissible in [22]. 
tower", using the terminology of [22].

This discussion motivates our presentation of rational term rewriting in Section 3, which is an adaptation to the rational case of the definitions and results in [7]. In the same section we also introduce the rewriting of $\mu$-terms, which is as straightforward as possible. The main result of the paper will show the soundness of the (parallel) rewriting of $\mu$-terms with respect to the reduction of possibly infinite, rational set of redexes in their unfolded rational term.

In Section 4 we provide a logical presentation of $\mu$-term rewriting and of rational rewriting. For the logical viewpoint, our starting point is the seminal work of José Meseguer about Rewriting Logic [25]. The basic idea is to consider a rewriting system $\mathcal{R}$ as a logical theory, and any rewriting as a sequent entailed by that theory. The entailment relation is defined inductively by suitable deduction rules, showing how sequents can be derived from other sequents. Sequents themselves are triples $\langle\alpha, t, s\rangle$, where $\alpha$ is an element of a so-called algebra of proof terms, encoding a justification of the rewriting of $t$ into $s$.

The original presentation of rewriting logic dealt with the finitary case. We consider here a variation of it, called (one-step) Preiteration Rewriting Logic, by introducing suitable rules for $\mu$-terms. The faithfulness of this presentation of $\mu$-term rewriting with respect to the original formulation is expressed by a result stating that there is bijection between sequents relating two terms and parallel reductions between them. The advantage of this logical approach is that not only the terms, but also the reductions are now endowed with an algebraic structure (the structure of proof terms), and this allows us to obtain a more precise relationship between $\mu$-term and rational rewriting with respect to the results in Section 3. In fact, we obtain a faithful (in the above sense) logical presentation of rational rewriting by considering rational sequents, i.e., equivalence classes of sequents with respect to suitable axioms.

Finally, in the concluding section we discuss the relationship with related papers, and we hint at some topics for future work.

\section{Rational Terms and $\mu$-terms}

The study of infinite terms is one of the most relevant contribution of computer science to the field of Universal Algebra. The starting point was the midSeventies work of the ADJ group (see e.g. $[19,18]$ ) on continuous algebras, which put the basis for the studies on varieties of ordered algebras, that is, algebras where the carrier is a partial order (see also [2]).

We assume the reader to be familiar with the usual notion of algebra over a signature $\Sigma$ (that is, a ranked alphabet of operator symbols $\Sigma=\cup_{n \in \mathbf{N}} \Sigma_{n}$, saying that $f$ is of arity $n$ for $f \in \Sigma_{n}$ ). We denote by $\Sigma$-Alg the category of algebras over $\Sigma$, and of $\Sigma$-homomorphisms. Continuous algebras are simply algebras where the carrier is not just a set, but rather a complete partial order, and the operators are continuous functions. Correspondingly, since homomorphisms must preserve the algebraic structure, they are required to be strict continuous functions. 
Definition 1 (complete partial orders). A partial order $\langle D, \leq\rangle$ is complete (is a CPO) if it has an element $\perp$ (called bottom) such that $\perp \leq d$ for all $d \in D$, and it has least upper bounds (LUB's) for all $\omega$-chains of elements. If $\left\{d_{i}\right\}_{i<\omega}$ is an $\omega$-chain (i.e., $d_{i} \leq d_{i+1}$ for all $i<\omega$ ), we denote its LUB by $\bigsqcup_{i<\omega}\left\{d_{i}\right\}$. A continuous function $f:\langle D, \leq D\rangle \rightarrow\left\langle D^{\prime}, \leq D^{\prime}\right\rangle$ between CPO's is a function $f: D \rightarrow D^{\prime}$ which preserves LUB's of $\omega$-chains, i.e., $f\left(\bigsqcup_{i<\omega}\right)\left\{d_{i}\right\}=\bigsqcup_{i<\omega}\left\{f\left(d_{i}\right)\right\}$; it is strict if $f\left(\perp_{D}\right)=\perp_{D^{\prime}}$. CPO denotes the category of CPO's and continuous functions.

We denote with $\Sigma$-CAlg the category of continuous algebras and strict continuous homomorphisms. We recall now the basic definitions and the main results on initial algebras and rational terms that will be used along the paper; these are borrowed from $[3,19,17]$, to which we refer the interested reader.

It is well-known that, for each signature $\Sigma$, the category $\Sigma$-Alg has an initial object, often called the word algebra and denoted by $T_{\Sigma}$. Its elements are all the terms freely generated from the constants and the operators of $\Sigma$, and can be regarded as finite trees whose nodes are labeled by operator symbols. As shown in [19], also the category $\Sigma$-CAlg has an initial object, denoted $C T_{\Sigma}$. Its elements are possibly infinite, possibly partial terms freely generated from $\Sigma$, and they form a CPO where the ordering relation is given by $t \leq t^{\prime}$ iff $t^{\prime}$ is "more defined" than $t$. We introduce directly $C T_{\Sigma}$, since $T_{\Sigma}$ can be recovered as a suitable sub-algebra: definitions are borrowed from [19], with minor changes.

Definition 2 (terms as functions). Let $\omega^{*}$ be the set of all finite strings of positive natural numbers; its elements are called occurrences, and the empty string is denoted by $\lambda$. Furthermore, let $\Sigma$ be a signature and $X$ be a set of variables such that $\Sigma \cap X=\emptyset$. A term over $(\Sigma, X)$ is a partial function $t: \omega^{*} \rightarrow$ $\Sigma \cup X$ such that the domain of definition of $t, \mathcal{O}(t)$, satisfies (for $w \in \omega^{*}$ and $i \in \omega)$

- $w i \in \mathcal{O}(t) \Rightarrow w \in \mathcal{O}(t)$

- wi $\in \mathcal{O}(t) \Rightarrow t(w) \in \Sigma_{n}$ for some $n \geq i$.

$\mathcal{O}(t)$ is called the set of occurrences of $t$. A term $t$ is total if $t(w) \in \Sigma_{n} \Rightarrow w i \epsilon$ $\mathcal{O}(t)$ for all $0<i \leq n ; t$ is finite if so is $\mathcal{O}(t)$; and $t$ is linear if no variable occurs more than once in it.

Given an occurrence $w \in \omega^{*}$ and a term $t \in C T_{\Sigma}(X)$, the subterm of $t$ at (occurrence) $w$ is the term $t / w$ defined as $t / w(u)=t(w u)$ for all $u \in \omega^{*}$.

The set of terms over $(\Sigma, X)$ is denoted by $C T_{\Sigma}(X)$, and $C T_{\Sigma}$ stays for $C T_{\Sigma}(\emptyset)$. For finite, total terms, this description is equivalent to the usual representation of terms as operators applied to other terms. Partial terms are made total in this representation by introducing the undefined term $\perp$, which represents the empty function $\perp: \emptyset \rightarrow \Sigma \cup X$, always undefined. Thus, for example, if $x \in X, t=f(\perp, g(x))$ is the term such that $\mathcal{O}(t)=\{\lambda, 2,2 \cdot 1\}, t(\lambda)=f \in \Sigma_{2}$, $t(2)=g \in \Sigma_{1}$, and $t(2 \cdot 1)=x \in X$.

$C T_{\Sigma}(X)$ forms a CPO with respect to the "approximation" relation. We say that $t$ approximates $t^{\prime}$ (written $t \leq t^{\prime}$ ) iff $t$ is less defined than $t^{\prime}$ as partial 
function. The least element of $C T_{\Sigma}(X)$ with respect to $\leq$ is clearly $\perp$. An $\omega$ chain $\left\{t_{i}\right\}_{i<\omega}$ is an infinite sequence of terms $t_{0} \leq t_{1} \leq \ldots$. Every $\omega$-chain $\left\{t_{i}\right\}_{i<\omega}$ in $C T_{\Sigma}(X)$ has a LUB $\bigcup_{i<\omega}\left\{t_{i}\right\}$ characterized as follows:

$$
t=\bigcup_{i<\omega}\left\{t_{i}\right\} \quad \Leftrightarrow \quad \forall w \in \omega^{*} \cdot \exists i<\omega . \forall j \geq i \cdot t_{j}(w)=t(w) .
$$

¿From $C T_{\Sigma}, T_{\Sigma}$ can be recovered as the subalgebra of finite, total terms. In the paper our main interest is in rational terms.

Definition 3 (rational terms). A term $t$ over $(\Sigma, X)$ is rational if the associated set of prefixes $\mathcal{P}(t)=\{\langle w, t(w)\rangle \mid w \in \mathcal{O}(t)\}$ is regular, that is, if it is recognizable from a finite automata. Equivalently, $t$ is rational if the set of all its subterms $\{t / u \mid u \in \mathcal{O}(t)\}$ is finite.

The collection of all rational terms over $(\Sigma, X)$ is denoted by $R T_{\Sigma}(X)$, and it is easily shown to be a subalgebra of $C T_{\Sigma}(X)$, but not a continuous one.

A different approach to the study of infinite terms, and in particular to the characterization of rational terms, focussed instead on the extension of the notion of signature by means of suitable recursion operators, and on an axiomatic characterization of unique fixed-points. A seminal stream (with tight links to the categorical notion of algebraic theories [24]) started with the paper on algebraic iterative theories by Elgot [13]. Here we recall just a few basic results, for which we refer the reader to [4].

Definition 4 ( $\mu$-terms). Let $\Sigma$ be a signature and $X$ be a (countably infinite) set of variables such that $\Sigma \cap X=\emptyset$. The set $\mu T_{\Sigma}(X)$ of $\mu$-terms over $(\Sigma, X)$ is defined as the smallest set of expressions satisfying the following clauses:

$-x \in \mu T_{\Sigma}(X) \quad$ if $x \in X$

$-f\left(t_{1}, \ldots, t_{n}\right) \in \mu T_{\Sigma}(X)$ if $f \in \Sigma_{n}, t_{i} \in \mu T_{\Sigma}(X)$;

$-\mu_{x} . t \in \mu T_{\Sigma}(X) \quad$ if $x \in X, t \in \mu T_{\Sigma}(X)$.

Equivalently, let $\Sigma_{X}^{\mu}=\Sigma \uplus\left\{\mu_{x} \mid x \in X\right\}$ be a signature that extends $\Sigma$ with one unary operator for each variable in $X$. Then $\mu$-terms over $(\Sigma, X)$ can also be defined as finite terms over $\Sigma_{X}^{\mu}$, i.e., elements of the word algebra $T_{\Sigma_{X}^{\mu}}(X)$.

Consistently with the interpretation described in the Introduction, operator $\mu_{x}$ is a binding operator for variable $x$. Thus we define the set of free variables $F V(t)$ for a term $t$ in the usual way, we call closed any term with no free variables, and we identify terms up to $\alpha$-conversion.

Substitutions are functions from variables to terms that, by freeness, can be extended in a unique way to operator preserving functions from terms to terms. Since we are dealing with two different kind of terms, we introduce now two types of substitutions which will be used in the sequel. 
Definition 5 (continuous and parameter substitutions). Let $\Sigma$ be a signature and $X, Y$ be two (countably infinite) sets of variables such that $\Sigma \cap X=$ $\Sigma \cap Y=\emptyset$. A (continuous) substitution from $X$ to $Y$ is a function $\sigma: X \rightarrow$ $C T_{\Sigma}(Y)$ (used in postfix notation). It uniquely determines a strict continuous $\Sigma$-homomorphism (also denoted by $\sigma$ ) from $C T_{\Sigma}(X)$ to $C T_{\Sigma}(Y)$, which extends $\sigma$ as follows:

$-\perp \sigma=\perp$

$-f\left(t_{1}, \ldots, t_{n}\right) \sigma=f\left(t_{1} \sigma, \ldots, t_{n} \sigma\right)$

- $\left(\bigcup_{i<\omega}\left\{t_{i}\right\}\right) \sigma=\bigcup_{i<\omega}\left\{t_{i} \sigma\right\}$.

A parameter substitution is a function $\sigma: X \rightarrow \mu T_{\Sigma}(X \cup Y)$. It uniquely determines an operator preserving function from $\mu T_{\Sigma}(X)$ to $\mu T_{\Sigma}(X \cup Y)$, as follows

$-x \sigma=\sigma(x)$

$-f\left(t_{1}, \ldots, t_{n}\right) \sigma=f\left(t_{1} \sigma, \ldots, t_{n} \sigma\right)$

$-\left(\mu_{x} . t\right) \sigma=\mu_{x} \cdot\left(t \sigma_{x}\right)$,

where $\sigma_{x}(y)=x$ if $x=y$, and $\sigma_{x}(y)=\sigma(y)$ otherwise.

A substitution is finite if there is only a finite number of variables $x$ such that $\sigma(x) \neq x$ : it will be described as a finite set $\left\{x_{1} / t_{1}, \ldots, x_{n} / t_{n}\right\}$ with $t_{i}=\sigma\left(x_{i}\right)$ for all $1 \leq i \leq n$.

As for classical algebras, exploiting the syntactical nature of $\mu$-terms one can define suitable structures where operators can be interpreted, called preiteration algebras [4]. For our purposes, it is enough to know that the set $\mu T_{\Sigma}(X)$ forms the free preiteration algebra over $X$ in the category $\Sigma$-PIAlg, where objects are preiteration algebras and arrows are preiteration homomorphisms, that is, homomorphisms preserving also the $\mu$ 's.

In this framework an equation is a pair $\langle t, s\rangle$ of $\mu$-terms, and the class of preiteration algebras satisfying an equational specification forms a suitable variety, a la Birkhoff. In particular, we are interested in the variety of iteration algebras, and more specifically in the free iteration algebra. Among the many equivalent axiomatizations of this free algebra, we prefer the following one (based actually on conditional equations) for its clarity and conciseness. Other presentations are described in [4], which also presents informal explanations for the rules below.

Definition 6 (free iteration algebra). Given a signature $\Sigma$ and a (countably infinite) set $X$ of variables, let $\cong$ be the least congruence relation over $\mu T_{\Sigma}(X)$, closed with respect to parameter substitutions, induced by the following rules

- (composition)

$$
\overline{\mu_{x} .(t\{x / s\})=t\left\{x / \mu_{x} \cdot(s\{x / t\})\right\}}
$$

- (left zero)

$$
\frac{x \notin F V(t)}{\mu_{x} . t=t}
$$


- (regularity)

$$
\frac{u \notin F V(t), \quad \mu_{u} \cdot(t\{x / u, y / u\})=\mu_{u} \cdot(s\{x / u, y / u\})}{\mu_{u} \cdot(t\{x / u, y / u\})=\mu_{y} \cdot\left(s\left\{x / \mu_{x} . t\right\}\right)} .
$$

We define the free iteration algebra over $(\Sigma, X)$ as the set $\mu T_{\Sigma}(X)_{/ \simeq}$, obtained by quotienting the free preiteration algebra $\mu T_{\Sigma}(X)$ by the congruence $\cong$.

As far as we know, Ginali in her Ph.D. thesis (see [17]) and independently Elgot, Bloom and Tindell [14] were the first to prove a correspondence result between the class of regular trees and Elgot's free iterative theories. Building on that result, Bloom and Ésik proved in [3] the following theorem.

Theorem 7 (rational terms and free iteration algebras). For any signature $\Sigma$ and set $X$ of variables, there is a preiteration isomorphism between the class $R T_{\Sigma}(X)$ of rational trees over $(\Sigma, X)$ and the class of elements of the free iteration algebra $\left.\mu T_{\Sigma}(X)\right) / \simeq$.

In the rest of the paper for a $\mu$-term $t$ we will denote by $[t]$ the rational term corresponding (via the isomorphism mentioned in the last result) to the equivalence class of $t$ modulo the axioms of Definition 6. Intuitively, $[t]$ is obtained as the limit of a chain of $\mu$-terms starting from $t$ and where at each step a suitable self-instantiation (via a parameter substitution) is applied. The only $\mu$-term to which this intuition is not immediately applicable is $\mu_{x} . x$ : the reader can safely assume that $\left[\mu_{x}, x\right]=\perp$ by definition.

\section{Rewriting of Rational Terms and of $\mu$-Terms}

The standard definition of term rewriting will be extended in this section to the rewriting of $\mu$-terms (i.e., closed elements of $\mu T_{\Sigma}(X)$ ) and of infinite terms (elements of $C T_{\Sigma}$ ) via finite rules. Borrowing from [7], besides the standard sequential derivations we will introduce an infinitary extension called infinite parallel rewriting which allows one to reduce infinitely many redexes of an infinite term in a single reduction step. In particular, we will focus on the subcase of rational rewriting, i.e., the parallel reduction of rational sets of redexes. The main result of the section will show the soundness of $\mu$-term rewriting with respect to rational term rewriting. Definitions and results are presented here for the class of orthogonal term rewriting systems only.

Definition 8 (term rewriting systems (TRS)). Let $X$ be a countably infinite set of variables. A term rewriting system $\mathcal{R}$ (over $X$ ) is a tuple $(\Sigma, L, R)$, where $\Sigma$ is a signature, ${ }^{4} L$ is a set of labels, and $R$ is a function $R: L \rightarrow$ $T_{\Sigma}(X) \times T_{\Sigma}(X)$, such that for all $d \in L$, if $R(d)=\langle l, r\rangle$ then $\operatorname{var}(r) \subseteq \operatorname{var}(l) \subseteq X$ and $l$ is not a variable.

A TRS $\mathcal{R}$ is orthogonal if all its rules are left-linear and non-overlapping, that is, the left-hand side of each rule does not unify with a non-variable subterm of any other rule in $\mathcal{R}$, or with a proper, non-variable subterm of itself.

\footnotetext{
${ }^{4}$ Often the signature will be understood.
} 
Given a term rewriting system (also TRS) $\mathcal{R}$, we usually write $d: l \rightarrow r \in R$ if $d \in L$ and $R(d)=\langle l, r\rangle$; to make explicit the variables contained in a rule, we write $d\left(x_{1}, \ldots, x_{n}\right): l\left(x_{1}, \ldots, x_{n}\right) \rightarrow r\left(x_{1}, \ldots, x_{n}\right) \in R$ where $\left\{x_{1}, \ldots, x_{n}\right\}=$ $\operatorname{var}(l)$. For example, the TRS $\mathcal{Z}=\left\{d: f(x, x) \rightarrow a, d_{1}: f(x, f(y, z)) \rightarrow a\right\}$ is not orthogonal: $d$ is not left-linear, while $f(x, f(y, z))$ can unify with its subterm $f(y, z)$.

The definitions below introduce the rewriting of infinite terms and of $\mu$-terms.

Definition 9 (subterm replacement). Given terms $t, s \in C T_{\Sigma}(X)$ and an occurrence $w \in \omega^{*}$, the replacement of $s$ in $t$ at (occurrence) $w$, denoted $t[w \leftarrow s]$, is the term defined as $t[w \leftarrow s](u)=t(u)$ if $w \& u$ or $t / w=\perp$, and $t[w \leftarrow$ $s](w u)=s(u)$ otherwise.

The definition of subterm replacement applies as it is to $\mu$-terms in $\mu T_{\Sigma}(X)$, simply considering them as finite terms over the extended signature $\Sigma_{X}^{\mu}$.

Definition 10 ((plain) redexes and $\mu$-redexes). Let $\mathcal{R}=\langle\Sigma, L, R\rangle$ be a TRS over $X$. A (plain) redex $\Delta$ of a term $t \in C T_{\Sigma}$ is a pair $\Delta=(w, d)$ where $w \in \omega^{*}$ is an occurrence, $d: l \rightarrow r \in R$ is a rule, and there exists a continuous substitution $\sigma: \operatorname{var}(l) \rightarrow C T_{\Sigma}$ such that $t / w=l \sigma$.

A $\mu$-redex $\Delta$ of a closed $\mu$-term $t \in \mu T_{\Sigma}(X)$ is a pair $\Delta=(w, d)$ where $w \in \omega^{*}$ is an occurrence, $d: l \rightarrow r \in R$ is a rule, and there exists a parameter substitution $\sigma: \operatorname{var}(l) \rightarrow \mu T_{\Sigma}(X)$ such that $t / w=l \sigma$.

Definition 11 (reduction and derivation). Let $d: l \rightarrow r \in R$ be a rule and $\Delta=(w, d)$ be a redex of $t$. The result of its application is $s=t[w \leftarrow r \sigma]$. We also write $t \rightarrow_{\Delta} s$, and we say that $t$ reduces to $s$ (via $\Delta$ ). We say that there is a derivation from $t$ to $t^{\prime}$ if there are redexes $\Delta_{1}, \ldots, \Delta_{n}$ such that $t \rightarrow_{\Delta_{1}} t_{1} \rightarrow_{\Delta_{2}}$ $\ldots \rightarrow \Delta_{n} t_{n}=t^{\prime}$.

The last definition applies both to plain and to $\mu$-redexes: simply, if $\Delta$ is a $\mu$-redex of $t$, bound variables in $t$ are not affected in some undesirable way thanks to the fact that the matching substitution is required to be a parameter substitution. In this case, sometimes we will denote the corresponding reduction by $t \stackrel{\mu}{\rightarrow}_{\Delta} s$.

Sequential term rewriting, as just defined, can be generalized to parallel term rewriting by allowing for the simultaneous application of two or more redexes to a term. The definitions below summarize those in [6] (see also [23, 7]), and are valid for orthogonal TRS's only: as for subterm replacement, all definitions and results lift smoothly to $\mu$-terms.

Definition 12 (residuals). Let $\Delta=(w, d)$ and $\Delta^{\prime}=\left(w^{\prime}, d^{\prime}: l^{\prime} \rightarrow r^{\prime}\right)$ be two redexes in a term $t$. The set of residuals of $\Delta$ by $\Delta^{\prime}$, denoted by $\Delta \backslash \Delta^{\prime}$, is defined as:

$$
\Delta \backslash \Delta^{\prime}= \begin{cases}\emptyset & \text { if } \Delta=\Delta^{\prime} ; \\ \{\Delta\} & \text { if } w \ngtr w^{\prime} ; \\ \left\{\left(w^{\prime} w_{x} u, d\right) \mid r^{\prime} / w_{x}=l^{\prime} / v_{x}\right\} & \text { if } w=w^{\prime} v_{x} u \text { and } l^{\prime} / v_{x} \text { is a variable. }\end{cases}
$$


Note that $\Delta \backslash \Delta^{\prime}$ can contain more than one redex, whenever the right-hand side of the rule $d^{\prime}$ is not linear. As an example, consider the TRS $\mathcal{W}=\{d$ : $\left.f(x) \rightarrow g(x, x), d^{\prime}: a \rightarrow b\right\}$ and the redexes $\Delta=\left(1, d^{\prime}\right), \Delta^{\prime}=(\lambda, d)$ in the term $f(a)$ : then $\Delta / \Delta^{\prime}=\left\{\left(1, d^{\prime}\right),\left(2, d^{\prime}\right)\right\}$.

Proposition 13 (residual of a reduction). Let $\Phi \cup\{\Delta\}$ be a finite set of redexes of $t$, such that $t \rightarrow \Delta s$. Then the set $\Phi \backslash \Delta$ of residuals of $\Phi$ by $\Delta$, defined as the union of $\Delta^{\prime} \backslash \Delta$ for all $\Delta^{\prime} \in \Phi$, is a set of redexes in $s$.

The well-definedness of the notions below is based on the previous result.

Definition 14 (residual of a sequence, complete development). Let $\Phi$ be a finite set of redexes of $t$ and $\rho=\left(t \rightarrow \Delta_{1} t_{1} \ldots \rightarrow_{\Delta_{n}} t_{n}\right)$ be a reduction sequence. Then $\Phi \backslash \rho$ is defined as $\Phi$ if $n=0$, and as $\left(\Phi \backslash \Delta_{1}\right) \backslash \rho^{\prime}$, where $\rho^{\prime}=$ $\left(t_{1} \rightarrow_{\Delta_{2}} t_{2} \ldots \rightarrow \Delta_{n} t_{n}\right)$, otherwise.

A development of $\Phi$ is a reduction sequence such that after each initial segment $\rho$, the next reduced redex is an element of $\Phi \backslash \rho$. A complete development of $\Phi$ is a development $\rho$ such that $\Phi \backslash \rho=\emptyset$.

Proposition 15 (uniqueness of complete developments). All complete developments $\rho$ and $\rho^{\prime}$ of a finite set of redexes $\Phi$ in a term $t$ are finite, and end with the same term. Moreover, for each redex $\Delta$ of $t$, it holds $\Delta \backslash \rho=\Delta \backslash \rho^{\prime}$. Therefore we can safely denote by $\Delta \backslash \Phi$ the residuals of $\Delta$ by any complete development of $\Phi$ (and similarly replacing $\Delta$ with a finite set of redexes $\Phi^{\prime}$ of $t$ ).

Exploiting this result (whose proof can be found in [6]), we define the parallel reduction of a finite set of redexes as any complete development of them.

Definition 16 (parallel reduction). Given a finite set $\Phi$ of redexes in a term $t$, we write $t \rightarrow_{\Phi} t^{\prime}$ and say that there is a parallel reduction from $t$ to $t^{\prime}$ if there exists a complete development $t \rightarrow_{\Delta_{1}} t_{1} \ldots \rightarrow_{\Delta_{n}} t^{\prime}$ of $\Phi$.

Thus parallel rewriting allows to reduce a finite set of redexes of a term in a single, parallel step. If we consider an infinite term, there might be infinitely many distinct redexes in it: since the simultaneous rewriting of any finite subset of those redexes is well-defined, by a continuity argument one would expect that also the simultaneous rewriting of infinitely many redexes in an infinite term can be properly defined. We present here a definition which makes use of a suitable limit construction: for details we refer to [7]. It is however worth noticing that since $\mu$-terms are finite by Definition 4 , this infinitary extension is meaningful for plain redexes only.

Definition 17 (infinite parallel reduction). Given an infinite set $\Phi$ of redexes in a term $t$, let $t_{0} \leq t_{1} \leq t_{2} \ldots$ be any chain of finite terms such that its LUB is $t$, and for each $i<\omega$, every redex $(w, d) \in \Phi$ is either a redex of $t_{i}$ or $t_{i}(w)=\perp$ (that is, the image of the left-hand side of every redex in $\Phi$ is either all in $t_{i}$, or it is outside, but does not "cross the boundary"). Let $\Phi_{i}$ be the subset 
of all redexes in $\Phi$ which are also redexes of $t_{i}$, and let $s_{i}$ be the result of the (finite) parallel reduction of $t_{i}$ via $\Phi_{i}$ (i.e., $t_{i} \rightarrow_{\Phi_{i}} s_{i}$ ). Then we say that there is an (infinite) parallel reduction from $t$ to $s \stackrel{\text { def }}{=} \bigcup_{i<\omega}\left\{s_{i}\right\}$ via $\Phi$, and we write $t \rightarrow_{\Phi} s$.

Let us consider the TRS $\mathcal{V}=\left\{d: f(x) \rightarrow g(x), d^{\prime}: g(x) \rightarrow x\right\}$. Then the infinite set of redexes $\Phi=1^{*} \times\{d\}=\{(\lambda, d),(1, d), \ldots\}$ can be applied to the infinite term $t=f^{\omega}=\bigcup_{i<\omega}\left\{f^{i}(\perp)\right\}$ : a suitable chain of finite approximations is given by $t_{i}=f^{i}(\perp)$, and the associated subset $\Phi_{i}$ is $\left\{\left(1^{j}, d\right) \mid j \leq i\right\}$. Then $t_{i} \rightarrow_{\Phi_{i}} g^{i}(\perp)$, and thus $t \rightarrow_{\Phi} g^{\omega}$ by definition. Next, the infinite set of redexes $\Phi^{\prime}=1^{*} \times\left\{d^{\prime}\right\}=\left\{\left(\lambda, d^{\prime}\right),\left(1, d^{\prime}\right), \ldots\right\}$ can be applied to $t^{\prime}=g^{\omega}$. Now a suitable chain approximating $g^{\omega}$ is $t_{i}^{\prime}=g^{i}(\perp)$, the associated subsets $\Phi_{i}^{\prime}$ are $\left\{\left(1^{j}, d^{\prime}\right) \mid j \leq\right.$ $i\}$, and clearly $t_{i}^{\prime} \rightarrow_{\Phi_{i}^{\prime}} \perp$. Therefore $g^{\omega} \rightarrow_{\Phi^{\prime}} \bigcup_{i<\omega} \perp=\perp$, which explains formally the reduction of the hypercollapsing tower described in the introduction.

The next result states that the reduction of an infinite set of redexes is a well-given definition.

Proposition 18 (infinite parallel reduction is well-defined). In the hypotheses of Definition 17:

1. For each $i<\omega, s_{i} \leq s_{i+1}$; i.e., $\left\{s_{i}\right\}_{i<\omega}$ is a chain.

2. Definition 17 is well-given; i.e., the result of the infinite parallel reduction of $t$ via $\Phi$ does not depend on the choice of the chain approximating $t$, provided that it satisfies the required conditions.

3. If the set $\Phi$ of redexes is finite, then the infinite parallel reduction of Definition 17 yields the same result as the parallel reduction of Definition 16.

¿From infinite parallel rewriting, rational rewriting can be easily recovered by suitably restricting the class of infinite sets of redexes which can be applied to a given rational term.

Definition 19 (rational term rewriting). Let $\mathcal{R}=\langle\Sigma, L, R\rangle$ be an orthogonal TRS over $X$, and let $\Sigma^{*}=\Sigma \uplus\left\{f^{*} \mid f \in \Sigma\right\}$ be an auxiliary signature. For a set of redexes $\Phi$ in a term $t$, the associated marked term $t_{\Phi}$ is a term over $\left(\Sigma^{*}, X\right)$ defined by the following clauses:

$$
t_{\Phi}(w)= \begin{cases}f^{*} & \text { if }(w, d) \in \Phi \text { and } t(w)=f \\ t(w) & \text { otherwise. }\end{cases}
$$

A set of redexes $\Phi$ of a rational term $t$ is rational if the associated marked term $t_{\Phi}$ is rational [21]. A parallel reduction $t \rightarrow_{\Phi} s$ is rational if so is $\Phi$.

Thus $t_{\Phi}$ is obtained by marking in $t$ all the operators which are root of a redex in $\Phi$. It is rather easy to prove that if $\Phi$ is a rational set of redexes of a term $t$ and $t \rightarrow_{\Phi} s$, then also $s$ is rational.

The main result of this section shows that the rewriting of $\mu$-terms is sound with respect to the rational rewriting of rational terms. 
Theorem 20 (soundness of $\mu$-rewriting w.r.t. rational rewriting). Let $\mathcal{R}$ be an orthogonal TRS.

(1) If $\Phi$ is a finite set of $\mu$-redexes of a $\mu$-term $t$ and $t \stackrel{\mu}{\rightarrow}_{\Phi} s$, then there is a rational set of redexes $\mathcal{U}(\Phi)$ such that $[t] \rightarrow \mathcal{U}(\Phi)[s]$.

(2) If $\Phi$ is a rational set of redexes of a term $t$, then there is a $\mu$-term $\mathcal{F}(t, \Phi)$ and a finite set of $\mu$-redexes $\mathcal{M}(t, \Phi)$ such that $[\mathcal{F}(t, \Phi)]=t, \mathcal{F}(t, \Phi) \stackrel{\mu}{\rightarrow}_{\mathcal{M}(t, \Phi)} s^{\prime}$, and $\left[s^{\prime}\right]=s$.

Proof outline. (1) The rational set of redexes $\mathcal{U}(\Phi)$ is determined by taking the marked $\mu$-term $t_{\Phi}$ (in the sense of Definition 19), by unfolding it obtaining the marked rational term $\left[t_{\Phi}\right]$, and by considering all redexes of $[t]$ whose root are in correspondence with the marked nodes of $\left[t_{\Phi}\right]$.

Next suppose that $[t] \rightarrow \mathcal{U}(\Phi) s^{\prime}$, i.e., according to Definition 17 , that there is a chain of finite terms $t_{0} \leq t_{1} \leq t_{2} \ldots$ having $[t]$ as LUB and satisfying suitable conditions with respect to $\Phi$, such that $t_{i} \rightarrow_{\Phi_{i}} s_{i}$ for all $i<\omega$, and $s^{\prime}=$ $\bigcup_{i<\omega}\left\{s_{i}\right\}$. Then it can be shown by induction that $s_{i} \leq[s]$ for all $i<\omega$, which implies $s^{\prime} \leq[s]$. For the converse, it must be shown (by the way in which the approximation ordering is defined) that for every occurrence $w$ such that $s^{\prime}(w)=\perp$, also $[s](w)=\perp$ holds. The only not obvious case here is when a $\perp$ is generated in $s^{\prime}$ by the reduction of a hypercollapsing tower, but this is shown to be possible only if a $\mu$-term equivalent to $\mu_{x} . x$ is generated in $s$ by the reduction of $\Phi$, which unfolds to $\perp$ in $[s]$.

(2) Since set $\Phi$ is rational, so is the marked term $t_{\Phi}$. A marked $\mu$-term $t^{\prime}$ is shown to exist, such that $\left[t^{\prime}\right]=t_{\Phi}$, and such that for each marked node there is a redex for its unmarked version, $\mathcal{F}(t, \Phi)$, having that node as root. ${ }^{5}$ Let then $\mathcal{M}(t, \Phi)$ be the set of such $\mu$-redexes of $\mathcal{F}(t, \Phi)$ : it is a rational set of redexes, and the rest of the statement holds by point (1).

Corollary 21. For an orthogonal TRS $\mathcal{R}$, the rewrite relation induced on rational terms by rational term rewriting of Definition 19 coincides with the rewrite relation induced by $\mu$-term rewriting, modulo the axioms of Definition 6 .

In our opinion, this result provides a completely satisfactory interpretation (or "semantics") of the rewriting of $\mu$-terms expressed via a suitable notion of rewriting of the corresponding unfoldings.

\section{Rational Rewriting, Algebraically}

In this section we introduce (one-step) preiteration and rational rewriting logic, exploiting the rewriting logic formalism proposed in [25] for reasoning in logical terms about rewriting. Such logics will be presented in the form of sequent calculi,

${ }^{5}$ For example, if $t=f^{\omega}, d: f(f(y)) \rightarrow g(y)$, and $\Phi=\left\{\left(1 \cdot(1 \cdot 1)^{i}, d\right) \mid i<\omega\right\}$, then $t_{\Phi}=f\left(f^{*}\left(f\left(f^{*}(\ldots)\right)\right)\right)$. In this case we cannot take $t^{\prime}=\mu_{x} . f\left(f^{*}(x)\right)$ (even if $\left[t^{\prime}\right]=t_{\Phi}$ ), because there is no redex rooted at $f^{*}$ (indeed, the redex would "cross" the $\mu$ operator), but we can take instead $t^{\prime}=f\left(\mu_{x} . f^{*}(f(x))\right)$. 
via deduction rules which allow to generate sequents. The one-step preiteration and rational rewriting logics are shown to specify sequents which are in oneto-one correspondence with $\mu$-terms and rational reductions, respectively. The added value of this approach is that not only the terms, but also the reductions are now endowed with an algebraic structure (using suitable proof terms), and this allows us to obtain a more precise relationship between $\mu$-term and rational rewriting with respect to Corollary 21 . Intuitively, using the notation of point (1) of Theorem 20, one would like to identify two sets of $\mu$-redexes $\Phi$ and $\Phi^{\prime}$ in equivalent (but distinct) $\mu$-terms $t$ and $t^{\prime}$ if the induced rational set of redexes coincide, i.e., if $\mathcal{U}(\Phi)=\mathcal{U}\left(\Phi^{\prime}\right)$. Interestingly, this can be obtained in the rewriting logic framework by providing the proof terms denoting $\mu$-term reductions with a pre-iteration structure, and by imposing on them exactly the same axioms of Definition 6.

Space constraints forbid us to introduce the deduction rules for sequential composition, which allow to derive sequents which model many-step reductions (as done for example in $[25,9]$ ). This will be included in the full version of the paper: we just discuss in the concluding section the relevance of this extension.

Definition 22 (rewriting sequents). Let $\mathcal{R}=\langle\Sigma, L, R\rangle$ be an orthogonal TRS over $X$. Let $\Lambda=\cup_{n} \Lambda_{n}$ be the signature containing all the rules $d: l \rightarrow r \in R$ with the corresponding arity given by the number of variables in $d$ : more precisely, for each $n, \Lambda_{n}=\left\{d \mid d\left(x_{1}, \ldots, x_{n}\right): l\left(x_{1}, \ldots, x_{n}\right) \rightarrow r\left(x_{1}, \ldots, x_{n}\right) \in R\right\}$. A proof term $\alpha$ is a $\mu$-term of the preiteration algebra $\mu T_{\mathcal{R}}(X)=\mu T_{\Sigma \cup \Lambda}(X)$ (we assume that there are no clashes of names between the two sets of operators). A (rewriting) sequent is a triple $\langle\alpha, t, s\rangle$ (usually written as $\alpha: t \rightarrow s$ ) where $\alpha$ is a proof term and $t, s \in \mu T_{\Sigma}(X)$.

A sequent is closed if the associated proof term is so. For a given term $t$ and a finite substitution $\left\{x_{1} / t_{1}, \ldots, x_{n} / t_{n}\right\}$, we usually write $t\left(t_{1}, \ldots, t_{n}\right)$ for $t \sigma$.

Definition 23 (one-step preiteration rewriting logic). Let $\mathcal{R}=\langle\Sigma, L, R\rangle$ be a TRS over $X$. We say that $\mathcal{R}$ entails the sequent $\alpha: t \rightarrow s$ if it can be obtained by a finite number of applications of the following rules of deduction:

- (reflexivity)

$$
\frac{x \in X}{x: x \rightarrow x}
$$

- (instantiation)

$$
\frac{d: l \rightarrow r \in R, \quad d \in \Lambda_{n}, \quad \alpha_{i}: t_{i} \rightarrow s_{i} \text { for } i=1, \ldots, n}{d\left(\alpha_{1}, \ldots, \alpha_{n}\right): l\left(t_{1}, \ldots, t_{n}\right) \rightarrow r\left(s_{1}, \ldots, s_{n}\right)}
$$

- (congruence)

$$
\frac{f \in \Sigma_{n}, \quad \alpha_{i}: t_{i} \rightarrow s_{i} \text { for } i=1, \ldots, n}{f\left(\alpha_{1}, \ldots, \alpha_{n}\right): f\left(t_{1}, \ldots, t_{n}\right) \rightarrow f\left(s_{1}, \ldots, s_{n}\right)}
$$


- (recursion)

$$
\frac{\alpha: t \rightarrow s, \quad x \in X}{\mu_{x} . \alpha: \mu_{x} . t \rightarrow \mu_{x} . s}
$$

The class of sequents entailed by $\mathcal{R}$ induces a set-theoretical rewrite relation over terms, simply obtained by dropping the proof term of a sequent. Rule reflexivity is self-explaining: it allows any variable to be rewritten into itself, that is, to play an idle rôle during a rewriting step. Both recursion and congruence state that the rewrite relation is also compatible with respect to the algebraic structure, since it is closed under contexts. Maybe, the most interesting rule is instantiation: first, it implies that the transition relation is stable, that is, it is closed under substitutions. But the associated sequent describes also the simultaneous execution of nested rewrites: two subterms matching the left-hand sides of two rules can be rewritten simultaneously, in parallel, provided they do not overlap (and this is always the case for orthogonal systems).

Proposition 24 (sequents and parallel $\mu$-term rewriting). Let $\mathcal{R}$ be an orthogonal TRS. (1) If $\mathcal{R}$ entails a closed sequent $\alpha: t \rightarrow s$, then there is a set of $\mu$-redexes $\hat{\Phi}(\alpha)$ such that $t \rightarrow_{\hat{\Phi}(\alpha)} s$ (according to the parallel rewriting of Definition 16).

(2) Viceversa, if $\Phi$ is a set of $\mu$-redexes of $t$ and $t \rightarrow_{\Phi} s$, then there is a closed proof term $\hat{\alpha}(\Phi)$ such that $\mathcal{R}$ entails the sequent $\hat{\alpha}(\Phi): t \rightarrow s$.

(3) Functions $\hat{\Phi}$ and $\hat{\alpha}$ are inverse to each other.

Exploiting Theorem 7, we could easily obtain a description of the rewriting of rational terms by considering "abstract" sequents of the form $\alpha:[t] \rightarrow[s]$ for each sequent $\alpha: t \rightarrow s$ entailed by a TRS $\mathcal{R}$. However, using Theorem 20 we could obtain a result relating such sequents with the reduction of rational sets of redexes that is weaker than the last proposition, because the bijective correspondence would not hold. To ensure such a bijection we need to consider proof terms as well modulo the axioms of iteration algebras.

Definition 25 (one-step rational rewriting logic). A rational sequent has the form $\alpha: t \rightarrow s$, where $\alpha$ is a rational proof term (i.e., a rational term in $R T_{\Sigma \cup \Lambda}(X)$ ), and $t, s \in R T_{\Sigma}(X)$. A TRS $\mathcal{R}$ entails the rational sequent $\alpha: t \rightarrow s$ if it entails a sequent $\alpha^{\prime}: t^{\prime} \rightarrow s^{\prime}$ (according to Definition 23) such that $\alpha=\left[\alpha^{\prime}\right]$, $t=\left[t^{\prime}\right]$, and $s=\left[s^{\prime}\right]$. A sequent is closed if so is its proof term.

This definition of rational sequent allows us to lift the result of Proposition 24 to rational rewriting.

Proposition 26 (rational sequents and rational rewriting). Let $\mathcal{R}$ be an orthogonal TRS. (1) If $\mathcal{R}$ entails a closed rational sequent $\alpha: t \rightarrow s$, then there is a rational set of redexes $\hat{\Phi}(\alpha)$ such that $t \rightarrow_{\hat{\Phi}(\alpha)} s$ (according to the rational rewriting of Definition 19). 
(2) Viceversa, if $\Phi$ is a rational set of redexes of $t$ and $t \rightarrow_{\Phi} s$, then there is a closed rational proof term $\hat{\alpha}(\Phi)$ such that $\mathcal{R}$ entails the rational sequent $\hat{\alpha}(\Phi): t \rightarrow s$.

(3) Functions $\hat{\Phi}$ and $\hat{\alpha}$ are inverse to each other.

\section{Discussion and Future Work}

The main result presented in this paper is the fact that the parallel rewriting of $\mu$-terms (defined in a very natural way) provides a faithful implementation for rational term rewriting, i.e., for the parallel reduction of a possibly infinite (but rational) set of redexes in a rational term.

Some notions introduced here should be compared with the corresponding ones in [20], even if the focus of the papers is different. The notion of $\mu$-term rewriting of $[20]$ is quite different from ours, firstly because rewriting is defined

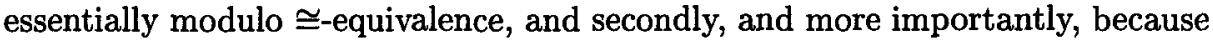
it is not allowed to rewrite a subterm $t^{\prime}$ of a $\mu$-term $t$ if $t^{\prime}$ contains a free variable which is bound in $t$. For example, rule $f(y) \rightarrow g(y)$ cannot be applied to the subterm $f(x)$ of $\mu_{x} . f(x)$. Furthermore, $\mu_{x} . x$ is not considered as a legal $\mu$-term. Such restrictions are motivated by the authors by potential problems that collapsing rules could cause. Recalling the discussion in the Introduction about the collapsing rule $g(y) \rightarrow y$, we can safely claim that such problems are due to the (implicit) use of the infinitary extension of term rewriting proposed in [22] as reference model for theory of $\mu$-term rewriting of the mentioned paper. In fact, such problems simply disappear using the theory of infinite parallel rewriting presented in [7], which provides a satisfactory interpretation for the $\mu$-term $\mu_{x} . x$, as well as for the reduction of hypercollapsing towers.

Closer to the soundness result of Section 3 are the adequacy results relating term graph rewriting and rational term rewriting proposed in [21] and [8]. In fact, possibly cyclic finite term graphs can be considered as an alternative finite representation of rational terms, where also "horizontal sharing" is allowed. In [21], the notion of adequacy between rewriting systems is introduced, which is essentially equivalent to soundness plus a form of partial completeness. ${ }^{6}$ In the same paper, it is presented an adequacy result between term graph rewriting and rational term rewriting defined using [22]; however, the result is restricted to the case of systems with at most one collapsing rules, or modulo hypercollapsing towers. In [8] instead, rational rewriting is defined exactly as in this paper, and it is shown that cyclic term graph rewriting using the algebraic approach is adequate for it, even in the presence of collapsing rules.

In the last section we showed essentially that the main result of the paper can be rephrased in a very elegant way by making explicit the algebraic structure of the one-step reductions (using proof terms). Recall that, by Theorem 7, rational

${ }^{6}$ As a concrete example, the result presented in Theorem 20 , which is actually stronger than a soundness result by point (2), could be rephrased as "parallel $\mu$-term rewriting is adequate for rational term rewriting". 
terms are $\cong$-equivalence classes of $\mu$-terms. Giving to one-step reductions of $\mu$ terms in an obvious way a $\mu$-term structure over a suitable signature, we are able to recover rational rewriting by imposing the congruence $\cong$ on proofs terms as well. In other words, the relationship between $\mu$-term and rational one-step rewriting is obtained simply by lifting the relationship between the corresponding class of terms to the level of reductions.

And one can go further, by lifting the same relationship to the level of rewriting sequences; due to space limitation the results we sketch here will appear in the full paper only. Full rewriting logic introduces a binary operator modeling sequential composition, and lifts the same algebraic structure of one-step reductions to whole derivations as well. The resulting structure provides a bridge between the standard presentation of rewriting and categorical models based on 2-categories as proposed for example in [26, 27], where arrows represent terms and cells represent rewriting sequences. As in the case of the "one-step" variants, we can consider both (full) preiteration and rational rewriting logic, and the corresponding categorical presentations based on preiteration and iteration 2-categories, respectively [5]. Furhtermore, it can be shown that they can be generated via a free construction from a suitable representation of a term rewriting systems as a suitable computad.

Finally, we mention that the formal framework just described, consisting in lifting the algebraic structure of terms to the level of reductions and of rewriting sequences and obtaining in this way categorical models, provides one interesting application of the general methodology for the semantics of structured transition systems proposed in [10].

\section{References}

1. A. Arnold and M. Nivat. The metric space of infinite trees. algebraic and topological properties. Fundamenta Informaticae, 4:445-476, 1980.

2. S. Bloom. Varieties of ordered algebras. Journal of Computer and System Science, 13:200-210, 1976.

3. S. Bloom and Z. Ésik. Iteration Theories. EATCS Monographs on Theoretical Computer Science. Springer Verlag, 1993.

4. S. Bloom and Z. Ésik. Solving polinomials fixed point equations. In Mathematical Foundations of Computer Science, volume 841 of $L N C S$, pages 52-67. Springer Verlag, 1994.

5. S.L. Bloom, Z. Ésik, A. Labella, and E.G. Manes. Iteration 2-theories. In Proceedings $A M A S T$ '97, 1997. To appear.

6. G. Boudol. Computational semantics of term rewriting systems. In M. Nivat and J. Reynolds, editors, Algebraic Methods in Semantics, pages 170-235. Cambridge University Press, 1985.

7. A. Corradini. Term rewriting in $C T_{\Sigma}$. In Proceedings $C A A P$ '93, volume 668 of LNCS, pages 468-484. Springer Verlag, 1993.

8. A. Corradini and F. Drewes. (Cyclic) term graph rewriting is adequate for rational parallel term rewriting. Technical Report TR-97-14, Dipartimento di Informatica, Pisa, 1997. 
9. A. Corradini and F. Gadducci. CPO Models for infinite term rewriting. In Algebraic Methodology and Software Technology, volume 936 of LNCS, pages 368-384. Springer Verlag, 1995.

10. A. Corradini and U. Montanari. An algebraic semantics for structured transition systems and its application to logic programs. Theoret. Comput. Sci., 103:51-106, 1992.

11. N. Dershowitz and S. Kaplan. Rewrite, rewrite, rewrite, rewrite, rewrite.... In Proc. POPL'89, Austin, pages 250-259, 1989.

12. N. Dershowitz, S. Kaplan, and D.A. Plaisted. Infinite normal forms (plus corrigendum). In Proc. ICALP'89, pages 249-262, 1989.

13. C. C. Elgot. Monadic computations and iterative algebraic theories. In Logic Colloquium 1973, volume 80 of Studies in Logic, pages 153-169. North Holland, 1975.

14. C.C. Elgot, C.C. Bloom, and R. Tindell. The algebraic structure of rooted trees. Journal of Computer and System Science, 16:362-339, 1978.

15. W.M. Farmer, J.D. Ramsdell, and R.J. Watro. A correctness proof for combinator reduction with cycles. ACM Trans. Program. Lang. Syst., 12:123-134, 1990.

16. W.M. Farmer and R.J. Watro. Redex capturing in term graph rewriting. In R.V. Book, editor, Proceedings of the 4th International Conference on Rewriting Techniques and Applications (RTA'91), volume 488 of LNCS, pages 13-24. Springer Verlag, 1991.

17. S Ginali. Regular trees and the free iterative theory. Journal of Computer and System Science, 18:222-242, 1979.

18. J.A. Goguen, J.W. Tatcher, E.G. Wagner, and J.R Wright. Some fundamentals of order-algebraic semantics. In Mathematical Foundations of Computer Science, volume 45 of $L N C S$, pages 153-168. Springer Verlag, 1976.

19. J.A. Goguen, J.W. Tatcher, E.G. Wagner, and J.R Wright. Initial algebra semantics and continuous algebras. Journal of the ACM, 24:68-95, 1977.

20. P. Inverardi and M. Venturini-Zilli. Rational rewriting. In Mathematical Foundations of Computer Science, volume 841 of LNCS, pages 433-442. Springer Verlag, 1994.

21. J.R. Kennaway, J.W. Klop, M.R. Sleep, and F.J. de Vries. On the adequacy of graph rewriting for simulating term rewriting. ACM Trans. Program. Lang. Syst., 16:493-523, 1994.

22. J.R. Kennaway, J.W. Klop, M.R. Sleep, and F.J. de Vries. Transfinite reductions in orthogonal term rewriting system. Information and Computation, 119:18-38, 1995.

23. C. Laneve and U. Montanari. Axiomatizing permutation equivalence in the $\lambda$ calculus. Mathematical Structures in Computer Science, 6:219-249, 1996.

24. F.W. Lawvere. Functorial semantics of algebraic theories. Proc. National Academy of Science, 50:869-872, 1963.

25. J. Meseguer. Conditional rewriting logic as a unified model of concurrency. Theoret. Comput. Sci., 96:73-155, 1992.

26. A.J. Power. An abstract formulation for rewrite systems. In Proceedings Category Theory in Computer Science, volume 389 of $L N C S$, pages 300-312. Springer Verlag, 1989.

27. D.E. Rydehard and E.G. Stell. Foundations of equational deductions: A categorical treatment of equational proofs and unification algorithms. In Proceedings Category Theory in Computer Science, volume 283 of LNCS, pages 114-139. Springer Verlag, 1987. 